\title{
Clinical impact of C-reactive protein to albumin ratio of the 7th postoperative day on prognosis after laparoscopic colorectal cancer surgery
}

Masahiro Kataoka ( $\square$ kataokamasa0926@gmail.com )

Suwa Red Cross Hospital

Kuniyuki Gomi

Suwa Red Cross Hospital

Ken Ichioka

Suwa Red Cross Hospital

Takuya Iguchi

Suwa Red Cross Hospital

Tomoki Shirota

Suwa Red Cross Hospital

Arano Makino

Suwa Red Cross Hospital

Ko Shimada

Suwa Red Cross Hospital

Kiyotomi Maruyama

Suwa Red Cross Hospital

Motohiro Mihara

Suwa Red Cross Hospital

Shoji Kajikawa

Suwa Red Cross Hospital

\section{Research Article}

Keywords: C-reactive protein to albumin ratio, colorectal cancer, curative resection, prognosis.

Posted Date: January 13th, 2022

DOI: https://doi.org/10.21203/rs.3.rs-1201094/v1

License: (c) (1) This work is licensed under a Creative Commons Attribution 4.0 International License.

Read Full License 


\section{Abstract \\ Background/Aim:}

C-reactive protein to albumin ratio (CAR) has been utilized as a prognostic factor in various carcinomas. We investigated the relationship between preoperative, first postoperative day (POD1), and seventh postoperative day (POD7) CARs and the prognosis of patients with colorectal cancer (CRC).

\section{Patients and Methods:}

320 patients with CRC who underwent laparoscopic radical resection between May 2011 and December 2016 were enrolled. Patients were selected into two groups, high CAR and low CAR, based on preoperative, POD1, and POD7 CARs. The relapse-free survival (RFS) and overall survival (OS) were compared between groups using propensity score matching.

\section{Results}

The high CAR group had a significantly worse RFS and OS ( $\mathrm{n}=72 /$ group, RFS: $p<0.001 ;$ OS: $p=0.002$ ) at POD7 than those in the low CAR group. However, in preoperative and POD1 analysis, no differences were observed.

\section{Conclusion}

In patients with colorectal cancer, CAR of POD7 was a significant prognostic factor.

\section{Background}

Colorectal cancer (CRC) is the third most common cancer and the second most common cause of cancerrelated death worldwide [1]. It is therefore essential to investigate the prognostic factors of CRC.

The influence of systemic inflammation in various carcinomas has been widely reported in recent years $[2,3]$. In addition, multiple biomarkers of systemic inflammation have been developed, including the prognostic nutritional index (PNI)[4], the neutrophil-to-lymphocyte ratio (NLR)[5], and the modified Glasgow prognostic score (mGPS) [6], all of which have been reported to be related to CRC prognosis. While mGPS uses C-reactive protein (CRP) and albumin levels, CRP to albumin ratio (CAR) is a calculated value developed by Fairclough et al. [7].

CAR has been reported to be useful in predicting disease outcomes such as sepsis [8], acute pancreatitis [9], and ulcerative colitis [10]. Furthermore, it has been reported to be a useful biomarker in various carcinomas, including gastric cancer [11], esophageal cancer [12], pancreatic cancer [13], gallbladder 
carcinoma [14], and ovarian cancer [15]. Both CRP and albumin, factors used in CAR, are synthesized in the liver. Chronic systemic inflammation induced by cancer cells increases CRP synthesis and decreases albumin synthesis in the liver. These two factors are indirect measures of systemic inflammation, metabolic abnormalities, and cachexia [16].

CAR has been associated with CRC prognosis $[17,18]$. However, the impact of perioperative CAR on the prognosis of colorectal cancer after surgery has not been thoroughly investigated. The purpose of this study is to evaluate the influence of preoperative and postoperative CAR on relapse-free survival rates (RFS) and overall survival rates (OS) for CRC patients who underwent radical resection.

\section{Patients And Methods}

Chart reviews were conducted retrospectively on 362 patients with CRC who underwent laparoscopic resection at Suwa Red Cross Hospital from May 2011 to December 2016. Patients with metastasis, residual tumor, recurrent $\mathrm{CRC}$, or synchronous malignancy were excluded. A total of 320 patients were included in the analysis. The study protocol was approved by the Ethics Committee of Suwa Red Cross Hospital.

Clinical variables, such as age, sex, the American Society of Anesthesiologists physical status score (ASA-PS), preoperative carcinoembryonic antigen (CEA) level, preoperative carbohydrate antigen (CA19-9) level, tumor information (location, size, histotype, presence of lymphatic or venous invasion, and stage classification), and postoperative complications (grade II or higher on the Clavien-Dindo classification) were obtained from historical data. The resected colorectal cancer was staged using the tumor-nodemetastasis (TNM) classification system of the 8th edition of the Union for International Cancer Control (UICC). Survival rate, recurrence status, and time to recurrence were also investigated. Serum CRP and albumin levels at preoperative, the first postoperative day (POD1), and the seventh postoperative day (POD7) were analyzed, and CAR was calculated by dividing the CRP level $(\mathrm{mg} / \mathrm{dl})$ by the albumin level $(\mathrm{g} / \mathrm{l})$.

We used EZR software (R Foundation for Statistical Computing, Vienna, Austria) for statistical processing. The cut-off values for each CAR at preoperative, POD1, and POD7 were determined by analyzing the receiver operating characteristic $(\mathrm{ROC})$ curves for survival rate. Patients were divided into two groups based on the cut-off value: the high CAR and low CAR groups. In addition, propensity score matching was used to minimize the bias between the two groups. We performed a 1:1 matching without replacement through nearest available matching, with caliper set at 0.2. Each patient was propensity scored, based on a logistic regression model addressing the following 11 covariates: age, sex, ASA-PS, serum CEA level, serum CA19-9 level, tumor location, tumor size, tumor histotype, lymphatic invasion, venous invasion, and TNM stage. Following matching, logistic regression analysis was performed to assess the difference in five-year RFS and five-year OS between the groups. Chi-square or Fisher's exact test tested differences in categorical variables, and survival rates (RFS, OS) were analyzed using the Kaplan-Meier method. Values of $p<0.05$ were considered significant. 


\section{Results}

Data of 320 patients with stage 0 - III CRC were collected for this study. With five-year overall survival as the endpoint, the optimal cut-off value for CAR was determined using the area under the curve (AUC) of the ROC curve. The preoperative cut-off value was 0.038 ( $A U C=0.666$ ), the cut-off value on POD1 was 2.007 (AUC=0.649), and the cut-off value on POD7 was 0.859 (AUC=0.632).

\section{Analysis of preoperative CAR}

First, we reviewed preoperative CAR. Before propensity score matching, there were 144 patients in the high CAR group (CAR $\geq 0.038$ ) and 176 patients in the low CAR group (CAR < 0.038). Patients in the high CAR group were older than those in the low CAR group (73.82 \pm 10.79 years vs. $68.62 \pm 11.83$ years; $p$ $<0.001)$ and had a higher proportion of males $(59.7 \% v s .46 .0 \% ; p=0.018)$. In addition, the high CAR group (vs. low CAR group) showed significant differences in ASA-PS $(p<0.001)$, preoperative CEA (CEA $\geq 5$ : $48.6 \%$ vs. $30.1 \% ; p=0.001)$, tumor size ( $\geq 50 \mathrm{~mm}$ : $47.2 \%$ vs. $15.3 \% ; p<0.001)$, lymphatic invasion $(54.9 \%$ vs. $40.9 \% ; p=0.014)$, venous invasion $(51.4 \%$ vs. $33.0 \% ; p=0.001)$, and pathological stage $(p<0.001)$. The two groups were then propensity score-matched in a 1:1 ratio ( $n=93 /$ group), and there were no differences between groups (Table 1). There was no significant difference in postoperative complications either before or after propensity score matching.

Before propensity score matching, the five-year RFS was $66.4 \%$ in the high CAR group compared to $82.7 \%$ in the low CAR group $(p<0.001)$. Also, five-year OS was $74.5 \%$ in the high CAR group and $89.5 \%$ in the low CAR group $(p<0.001)$ (Figure. $1 \mathrm{~A}$ and $\mathrm{B})$. After propensity score matching, the results showed that fiveyear RFS was $73.3 \%$ in the high CAR group and $76.1 \%$ in the low CAR group ( $p=0.784)$. The 5 -year OS was $83.0 \%$ in the High CAR group and $85.7 \%$ in the Low CAR group ( $p=0.669)$ (Figure. 1 C and D).

\section{Analysis of CAR on POD1}

Second, we reviewed CAR of POD1. Before propensity score matching, there were 95 patients in the high CAR group (CAR $\geq 2.007)$ and 225 patients in the low CAR group (CAR <2.007). The high CAR group (vs. low CAR group) showed significant differences in age (74.03 \pm 11.19 vs. $69.67 \pm 11.61 ; p=0.002)$, ASA-PS $(p<0.001)$, preoperative CEA (CEA $\geq 5$ : $51.6 \%$ vs. $32.9 \%$; $p=0.002)$, tumor size ( $\geq 50 \mathrm{~mm}: 43.2 \%$ vs. $24.0 \%$; $p=0.001)$, lymphatic invasion ( $60.0 \%$ vs. $41.8 \%$; $p=0.003)$, venous invasion $(52.6 \% v s .36 .4 \% ; p=0.009)$, and pathologic stage $(p=0.026)$. Next, the two groups were propensity score matched in a 1:1 ratio ( $n=81 /$ group), but no differences were observed between groups (Table 2). For postoperative complications, there was a significant difference even after propensity score matching.

Before propensity score matching, the five-year RFS was $62.7 \%$ in the high CAR group and $80.5 \%$ in the low CAR group $(p<0.001)$. Five-year OS was $68.4 \%$ in the high CAR group and $88.5 \%$ in the low CAR group $(p<0.001)$ (Figure. 2A and B). After propensity score matching, five-year RFS was $66.6 \%$ in the high CAR group and $71.5 \%$ in the low CAR group $(p=0.220)$. Five-year OS was $70.1 \%$ in the high CAR group and $83.4 \%$ in the low CAR group $(p=0.059)$ (Figure. $2 \mathrm{C}$ and $\mathrm{D})$. 


\section{Analysis of CAR on POD7}

Finally, we reviewed the CAR of POD7. Before propensity score matching, there were 75 patients in the high CAR group (CAR $\geq 0.859)$ and 245 patients in the low CAR group (CAR < 0.859). The high CAR group

(vs. Iow CAR group) was older (74.32 \pm 9.64 vs. $69.94 \pm 12.02 ; p=0.004)$, consisted of a higher proportion of males $(69.3 \%$ vs. $46.9 \% ; p=0.001)$, and showed significant difference in ASA-PS $(p<0.001)$. The two groups were then propensity score matched at a ratio of 1:1, and the number of patients in both groups was 72 each, with no significant differences in any of the measured variables (Table 3). There was a significant difference in postoperative complications even after propensity score matching.

Before propensity score matching, the five-year RFS was $50.0 \%$ in the high CAR group and $82.6 \%$ in the low CAR group $(p<0.001)$. Five-year OS was $63.8 \%$ in the high CAR group and $88.1 \%$ in the low CAR group $(p<0.001)$ (Figure. $3 \mathrm{~A}$ and B). After propensity score matching, five-year RFS was $49.6 \%$ in the High CAR group and $79.6 \%$ in the low CAR group $(p<0.001)$. Five-year OS was $64.0 \%$ in the high CAR group and $84.6 \%$ in the Low CAR group ( $p=0.002)$ (Figure. 3 C and $D$ ).

Out of the 144 patients selected by propensity score matching from POD7 results, an additional comparison was made between patients with postoperative complications (19 patients) and patients without postoperative complications (125 patients). The five-year RFS was $65.2 \%$ for patients with postoperative complications and $65.0 \%$ for patients without postoperative complications $(p=0.989)$. Fiveyear OS was $72.4 \%$ in patients with postoperative complications and $75.0 \%$ in patients without postoperative complications $(p=0.964)$ (Figure. $4 \mathrm{~A}$ and $\mathrm{B})$. The results showed that the presence of postoperative complications did not affect survival rate.

As shown above, before propensity score matching, all CARs of preoperative, POD1, and POD7 were associated with prognosis. However, after propensity score matching and background equalization, only the CAR of POD7 was found to have a significant relationship with the prognosis of CRC patients.

\section{Discussion}

Various studies have shown that biomarkers such as PNI and NLR, which reflect systemic inflammation, are prognostic factors for malignancy $[4,5]$. The mGPS, derived from serum albumin and CRP levels, is among these biomarkers and is predictive of prognosis in CRC patients [6]. CAR, which is calculated from the ratio of serum albumin to $\mathrm{C}$-reactive protein, has also been used as a prognostic marker. Both albumin and CRP in serum are proteins synthesized in the liver. In the presence of inflammation, CRP synthesis is stimulated by inflammatory cytokines, while albumin synthesis is suppressed [19]. CAR therefore sensitively detects systemic inflammation, and preoperative CAR has been reported to have prognostic utility in gastric cancer [20], esophageal cancer [21], and gallbladder carcinoma [14].

In this study, there was no clear interrelationship between preoperative and POD1 CAR and prognosis after propensity score matching. In contrast, CAR of POD7 showed a strong relationship with prognosis 
even after propensity score matching and was regarded as an independent factor. Although it is not clear why these results were obtained, several factors may be involved.

It is conceivable that one of the factors explaining the temporal difference in the relationship between CAR and perioperative timing may be postoperative complications. It has been previously reported that postoperative complications in CRC surgery are associated with a poorer prognosis [22]. Complications within the first week after surgery may cause a prolonged inflammatory response reflected in the CAR of POD7. In reality, however, the analysis showed that postoperative complications were not related to prognosis in this case group, and the effect was not significant. Therefore, other factors may have been present.

One possible factor is that CAR reflects the postoperative systemic inflammation caused by the residual tumor cells. Malignant tumors are known to produce inflammatory cytokines [23]. It has been reported that the local immune response and systemic inflammation caused by malignant tumors affect the survival rate of cancer patients [2]. Residual cancer cells may prolong the postoperative inflammatory response, resulting in a poor prognosis for patients. However, it is unclear how microscopic cancer cells in patients who have undergone curative resection will affect the body, and it is questionable whether they can cause a prolonged inflammatory state.

On the other hand, there is a report that surgical stress and postoperative acute phase reactants may promote tumor survival and growth after tumor resection [23]. It has also been reported that surgical stress increases the expression of E-selectin in vascular endothelial cells, which in turn may increase postoperative hematogenous metastasis [24]. According to these reports, the inflammation itself worsens the prognosis by creating an environment more conducive to cancer metastasis and growth. The impact of high invasiveness of surgery on the prognosis of patients cannot be denied, and reducing the invasiveness may improve the prognosis of patients.

The impact of high levels of inflammatory markers on POD7 on recurrence of malignancy has been reported elsewhere. Hayama et al. reported that the neutrophil-to-lymphocyte ratio of POD7 was a significant predictor of reduced RFS in stage II postoperative CRC patients [25]. In esophageal cancer, Ibuki et al. reported that RFS was significantly reduces when CRP levels were high on POD7 [26]. As shown in these studies and the present study, postoperative inflammation is associated with recurrence and prognosis, and therefore, merits further investigation.

Our study has several limitations. First, this is a retrospective, single-center study. Although propensity score matching has been performed, the possibility of random error, selection bias, and confounding cannot be ruled out. A multicenter, prospective study would strengthen the level of evidence. Second, there is no consensus cut-off value for CAR, which makes it difficult to apply clinically at this point. In this study, we used the ROC curve to determine the cut-off values for each day, but it may be necessary to calculate these values in a more extensive study. Finally, it is unclear whether any intervention in patients with high POD7 CAR would improve their prognosis. Further research is needed to address this critical point. 


\section{Conclusion}

The results of propensity score matching showed that CAR on POD7 was an independent prognostic factor for RFS and OS in patients with CRC after radical resection; however, no relationship was found for preoperative or POD1 CAR. Patients with high CAR on POD7 may require adjuvant chemotherapy and more careful management.

\section{Abbreviations}

CAR: C-reactive protein to albumin ratio; POD1: first postoperative day; POD7: seventh postoperative day; CRC: colorectal cancer; RFS: relapse-free survival; OS: overall survival; PNI: prognostic nutritional index; NLR: neutrophil-to-lymphocyte ratio; mGPS: modified Glasgow prognostic score; CRP: C-reactive protein; CAR: C-reactive protein to albumin ratio; ASA-PS: American Society of Anesthesiologists physical status score; CEA: carcinoembryonic antigen; CA19-9: carbohydrate antigen; TNM: tumor-node-metastasis classification system; UICC: The Union for International Cancer Control; ROC: receiver operating characteristic; AUC: area under the curve.

\section{Declarations}

\section{Acknowledgements}

The Authors wish to thank BioMed Proofreading LLC for English copyediting.

\section{Author's Contributions}

MK drafted the manuscript. KG supervised and reviewed its content. KI, TI, TS, AM, KS, KM, MM and SK reviewed the content as well. All authors have read and approved the final deliverable.

\section{Funding}

There are no funding resources to be reported or declared.

\section{Availability of data and materials}

The datasets used and/or analysed during the current study available from the corresponding author on reasonable request.

\section{Ethics approval and consent to participate}

This study was performed in accordance with the ethical standards of the Declaration of Helsinki (1964) and its subsequent amendments. This article was approved by ethics committee of Suwa Red Cross Hospital. Given the retrospective design, a waiver of participant consent was granted by the ethics committee. 


\section{Consent for publication}

Not applicable.

\section{Competing interests}

The authors declare that they have no competing interests.

\section{References}

1. Bray F, Ferlay J, Soerjomataram I, Siegel RL, Torre LA, Jemal A. Global cancer statistics 2018 : GLOBOCAN estimates of incidence and mortality worldwide for 36 cancers in 185 countries. CA Cancer J Clin. 2018;68(6):394-424.

2. Diakos $\mathrm{Cl}$, Charles KA, McMillan DC, Clarke SJ. Cancer-related inflammation and treatment effectiveness. Lancet Oncol. 2014;15(11):e493-503.

3. Candido J, Hagemann T. Cancer-related inflammation. J Clin Immunol. 2013;33 Suppl 1:S79-84.

4. Luvián-Morales J, González-Trejo S, Carrillo JF, Herrera-Goepfert R, Aiello-Crocifoglio V, GallardoRincón D, Ochoa-Carrillo FJ, Oñate-Ocaña LF. Association of the prognostic nutritional index and overall survival in patients with colorectal cancer: A STROBE compliant retrospective cohort study. Cancer Med. 2019;8(7):3379-88.

5. Haram A, Boland MR, Kelly ME, Bolger JC, Waldron RM, Kerin MJ. The prognostic value of neutrophilto-lymphocyte ratio in colorectal cancer: A systematic review. J Surg Oncol. 2017;115(4):470-9.

6. Numata K, Ono Y, Toda S, Kamioka Y, Suematsu H, Sawazaki S, Tsuchida K, Higuchi A, Saeki H, Rino $Y$ et al. Modified Glasgow Prognostic Score and Carcinoembryonic Antigen Predict Poor Prognosis in Elderly Patients with Colorectal Cancer. Oncol Res Treat. 2020;43(4):125-33.

7. Fairclough E, Cairns E, Hamilton J, Kelly C. Evaluation of a modified early warning system for acute medical admissions and comparison with C-reactive protein/albumin ratio as a predictor of patient outcome. Clin Med (Lond). 2009;9(1):30-3.

8. Ranzani OT, Zampieri FG, Forte DN, Azevedo LC, Park M. C-reactive protein/albumin ratio predicts 90day mortality of septic patients. PLoS One. 2013;8(3):e59321.

9. Kaplan M, Ates I, Akpinar MY, Yuksel M, Kuzu UB, Kacar S, Coskun O, Kayacetin E. Predictive value of C-reactive protein/albumin ratio in acute pancreatitis. Hepatobiliary Pancreat Dis Int. 2017;16(4):42430.

10. Sayar S, Kurbuz K, Kahraman R, Caliskan Z, Atalay R, Ozturk O, Doganay HL, Ozdil K. A practical marker to determining acute severe ulcerative colitis: CRP/albumin ratio. North Clin Istanb. 2020;7(1):49-55.

11. Yue L, Lu Y, Li Y, Wang Y. Prognostic Value of C-Reactive Protein to Albumin Ratio in Gastric Cancer: A Meta-Analysis. Nutr Cancer. 2020:1-8. 
12. Tamagawa H, Aoyama T, Tamagawa A, Komori K, Maezawa Y, Kano K, Murakawa M, Atsumi Y, Hara $\mathrm{K}$, Kazama K et al. Influence of the Preoperative C-Reactive Protein-to-Albumin Ratio on Survival and Recurrence in Patients With Esophageal Cancer. Anticancer Res. 2020;40(4):2365-71.

13. Vujic J, Marsoner K, Wienerroither V, Mischinger HJ, Kornprat P. The Predictive Value of the CRP-toAlbumin Ratio for Patients With Pancreatic Cancer After Curative Resection: A Retrospective Single Center Study. In Vivo. 2019;33(6):2071-8.

14. Utsumi M, Aoki $H$, Nagahisa $S$, Nishimura $S$, Une $Y$, Kimura $Y$, Watanabe $M$, Taniguchi $F$, Arata $T$, Katsuda K et al. Preoperative C-Reactive Protein/Albumin Ratio as a Predictive Factor for Gallbladder Carcinoma. In Vivo. 2020;34(4):1901-8.

15. Liu Y, Chen S, Zheng C, Ding M, Zhang L, Wang L, Xie M, Zhou J. The prognostic value of the preoperative c-reactive protein/albumin ratio in ovarian cancer. BMC Cancer. 2017;17(1):285.

16. McMillan DC. Systemic inflammation, nutritional status and survival in patients with cancer. Curr Opin Clin Nutr Metab Care. 2009;12(3):223-6.

17. Shibutani M, Maeda K, Nagahara H, Iseki Y, Ikeya T, Hirakawa K. Prognostic Significance of the Preoperative Ratio of C-Reactive Protein to Albumin in Patients with Colorectal Cancer. Anticancer Res. 2016;36(3):995-1001.

18. Shibutani M, Maeda K, Nagahara H, Iseki Y, Hirakawa K, Ohira M. The significance of the C-reactive protein to albumin ratio as a marker for predicting survival and monitoring chemotherapeutic effectiveness in patients with unresectable metastatic colorectal cancer. Springerplus. 2016;5(1):1798.

19. Gabay C, Kushner I. Acute-phase proteins and other systemic responses to inflammation. N Engl J Med. 1999;340(6):448-54.

20. Toiyama Y, Shimura T, Yasuda H, Fujikawa H, Okita Y, Kobayashi M, Ohi M, Yoshiyama S, Hiro J, Araki T et al. Clinical Burden of C-Reactive Protein/Albumin Ratio Before Curative Surgery for Patients with Gastric Cancer. Anticancer Res. 2016;36(12):6491-8.

21. Ishibashi Y, Tsujimoto H, Hiraki S, Kumano I, Yaguchi Y, Horiguchi H, Nomura S, Ito N, Shinto E, Aosasa $\mathrm{S}$ et al. Prognostic Value of Preoperative Systemic Immunoinflammatory Measures in Patients with Esophageal Cancer. Ann Surg Oncol. 2018;25(11):3288-99.

22. Arnarson Ö, Butt-Tuna S, Syk I. Postoperative complications following colonic resection for cancer are associated with impaired long-term survival. Colorectal Dis. 2019;21(7):805-15.

23. Miki C, Konishi N, Ojima E, Hatada T, Inoue Y, Kusunoki M. C-reactive protein as a prognostic variable that reflects uncontrolled up-regulation of the IL-1-IL-6 network system in colorectal carcinoma. Dig Dis Sci. 2004;49(6):970-6.

24. Hagi T, Kurokawa Y, Kobayashi N, Takahashi T, Saito T, Yamashita K, Tanaka K, Makino T, Yamasaki $\mathrm{M}$, Nakajima $\mathrm{K}$ et al. Anti-metastatic effect of methylprednisolone targeting vascular endothelial cells under surgical stress. Sci Rep. 2021;11(1):6268.

25. Hayama T, Hashiguchi Y, Okada Y, Ono K, Nemoto K, Shimada R, Ozawa T, Toyoda T, Tsuchiya T, linuma $\mathrm{H}$ et al. Significance of the 7th postoperative day neutrophil-to-lymphocyte ratio in colorectal 
cancer. Int J Colorectal Dis. 2020;35(1):119-24.

26. Ibuki Y, Hamai Y, Hihara J, Emi M, Taomoto J, Furukawa T, Yamakita I, Kurokawa T, Okada M. Role of Postoperative C-Reactive Protein Levels in Predicting Prognosis After Surgical Treatment of Esophageal Cancer. World J Surg. 2017;41(6):1558-65.

\section{Tables}

Table 1 Clinicopathological parameters of CRC patients with respect to preoperative CAR 
Variable Before PSM

\begin{tabular}{cllllll} 
& $\begin{array}{l}\text { High CAR } \\
(>0.038)\end{array}$ & $\begin{array}{l}\text { Low CAR } \\
(<0.038)\end{array}$ & $\begin{array}{l}p \text { - } \\
\text { Value }\end{array}$ & $\begin{array}{l}\text { High CAR } \\
(>0.038)\end{array}$ & $\begin{array}{l}\text { Low CAR } \\
(<0.038)\end{array}$ & $\begin{array}{c}p \text { Value } \\
\text { Sex, } \mathrm{n}\end{array}$ \\
\hline Male & 144 & 176 & & 93 & 93 & \\
\hline Female & $56(59.7 \%)$ & $81(46.0 \%)$ & & $53(57.0 \%)$ & $53(57.0 \%)$ & \\
\hline Age, yr & $73.83 \pm 10.79$ & $68.62 \pm 11.83$ & $<0.001$ & $71.94 \pm 11.76$ & $71.39 \pm 10.50$ & 0.738 \\
\hline ASA-PS & & & & & & \\
\hline 1 & $7(4.9 \%)$ & $25(14.2 \%)$ & & $7(7.5 \%)$ & $2(2.2 \%)$ & \\
\hline 2 & $87(60.4 \%)$ & $124(70.5 \%)$ & & $58(62.4 \%)$ & $69(74.2 \%)$ & \\
\hline 3 & $49(34.0 \%)$ & $27(15.3 \%)$ & & $27(29.0 \%)$ & $22(23.7 \%)$ & \\
\hline 4 & $1(0.7 \%)$ & $0(0.0 \%)$ & $<0.001$ & $1(1.1 \%)$ & $0(0.0 \%)$ & 0.114
\end{tabular}

CEA

$<5$

$>5$

$74(51.4 \%)$

$123(69.9 \%)$

$70(48.6 \%)$
$51(54.8 \%)$

$42(45.2 \%)$
$55(59.1 \%)$

$38(40.9 \%)$

0.657

CA19-9

$<35$

$114(79.2 \%) \quad 152(86.4 \%)$

$76(81.7 \%) \quad 74(79.6 \%)$

$>35$

$30(20.8 \%)$

$24(13.6 \%)$

0.100

$17(18.3 \%)$

$19(20.4 \%)$

0.853

Tumor location

\begin{tabular}{lllllll} 
Right-side & $52(36.1 \%)$ & $73(41.5 \%)$ & & $34(36.6 \%)$ & $34(36.6 \%)$ & \\
\hline Left-side & $92(63.9 \%)$ & $103(58.5 \%)$ & 0.358 & $59(63.4 \%)$ & $59(63.4 \%)$ & 1.000
\end{tabular}

Tumor size

\begin{tabular}{lllllll}
$<50 \mathrm{~mm}$ & $76(52.8 \%)$ & $149(84.7 \%)$ & & $69(74.2 \%)$ & $68(73.1 \%)$ & \\
\hline$>50 \mathrm{~mm}$ & $68(47.2 \%)$ & $27(15.3 \%)$ & $<0.001$ & $24(25.8 \%)$ & $25(26.9 \%)$ & 1.000
\end{tabular}

Tumor histotype

\begin{tabular}{lllllll} 
pap/tub & $133(92.4 \%)$ & $170(96.6 \%)$ & & $89(95.7 \%)$ & $87(93.5 \%)$ & \\
\hline muc/por/sig & $11(7.6 \%)$ & $6(3.4 \%)$ & 0.132 & $4(4.3 \%)$ & $6(6.5 \%)$ & 0.747
\end{tabular}

Lymphatic

invasion

No

$65(45.1 \%) \quad 104(59.1 \%)$

50 (53.8\%)

47 (50.5\%) 

Yes
79 (54.9\%)
$72(40.9 \%)$
0.014
$43(46.2 \%)$
46 (49.5\%)
0.769

Venous invasion

\begin{tabular}{lllllll}
\hline No & $70(48.6 \%)$ & $118(67.0 \%)$ & & $53(57.0 \%)$ & $54(58.1 \%)$ & \\
\hline Yes & $74(51.4 \%)$ & $58(33.0 \%)$ & 0.001 & $40(43.0 \%)$ & $39(41.9 \%)$ & 1.000
\end{tabular}

Pathological stage

\begin{tabular}{|c|c|c|c|c|c|c|}
\hline 0 & $5(3.5 \%)$ & $29(16.5 \%)$ & & 5 (5.4\%) & 7 (7.5\%) & \\
\hline I & $24(16.7 \%)$ & $56(31.8 \%)$ & & $24(25.8 \%)$ & $23(24.7 \%)$ & \\
\hline II & $68(47.2 \%)$ & 45 (25.6\%) & & 35 (37.6\%) & 30 (32.3\%) & \\
\hline III & $47(32.6 \%)$ & $46(26.1 \%)$ & $<0.001$ & 29 (31.2\%) & 33 (35.5\% & 0.805 \\
\hline \multicolumn{7}{|l|}{$\begin{array}{l}\text { Postoperative } \\
\text { complication }\end{array}$} \\
\hline $\begin{array}{l}\text { Clavien-Dindo } \\
>\text { grade II }\end{array}$ & $13(9.1 \%)$ & 12 (6.9\%) & 0.533 & 7 (7.6\%) & 9 (9.8\%) & 0.795 \\
\hline
\end{tabular}

CRC, Colorectal cancer; CAR, C-reactive protein/albumin ratio; PSM, propensity score matching; ASAPS, American Society of Anesthesiologists physical status

CEA, carcinoembryonic antigen; CA19-9, carbohydrate antigen; pap, papillary adenocarcinoma; tub, tubular adenocarcinoma; muc, mucinous adenocarcinoma

por, poorly differentiated adenocarcinoma; sig, signet-ring cell carcinoma

Table 2 Clinicopathological parameters of CRC patients with respect to CAR at the 1st postoperative day 
Variable Before PSM

\begin{tabular}{|c|c|c|c|c|c|c|}
\hline & $\begin{array}{l}\text { High CAR } \\
(>2.007)\end{array}$ & $\begin{array}{l}\text { Low CAR } \\
(<2.007)\end{array}$ & $\begin{array}{l}p- \\
\text { Value }\end{array}$ & $\begin{array}{l}\text { High CAR } \\
(>2.007)\end{array}$ & $\begin{array}{l}\text { Low CAR } \\
(<2.007)\end{array}$ & $\stackrel{p}{\text { Value }}$ \\
\hline Sex, n & 95 & 225 & & 81 & 81 & \\
\hline Male & 45 (47.4\%) & $122(54.2 \%)$ & & 43 (53.1\%) & $43(53.1 \%)$ & \\
\hline Female & $50(52.6 \%)$ & $103(45.8 \%)$ & 0.273 & 38 (46.9\%) & 38 (46.9\%) & 1.000 \\
\hline Age, yr & $74.03 \pm 11.19$ & $69.67 \pm 11.61$ & 0.002 & $72.36 \pm 11.07$ & $73.47 \pm 10.40$ & 0.511 \\
\hline \multicolumn{7}{|l|}{ ASA-PS } \\
\hline 1 & $7(7.4 \%)$ & $25(11.1 \%)$ & & $7(8.6 \%)$ & $7(8.6 \%)$ & \\
\hline 2 & $50(52.6 \%)$ & $161(71.6 \%)$ & & $45(55.6 \%)$ & 48 (59.3\%) & \\
\hline 3 & 38 (40.0\%) & 38 (16.9\%) & & $29(35.8 \%)$ & $25(30.9 \%)$ & \\
\hline 4 & $0(0.0 \%)$ & $1(0.4 \%)$ & $<0.001$ & $0(0.0 \%)$ & $1(1.2 \%)$ & 0.813 \\
\hline \multicolumn{7}{|l|}{ CEA } \\
\hline$<5$ & $46(48.4 \%)$ & 151 (67.1\%) & & $44(54.3 \%)$ & $48(59.3 \%)$ & \\
\hline$>5$ & 49 (51.6\%) & $74(32.9 \%)$ & 0.002 & 37 (45.7\%) & $33(40.7 \%)$ & 0.634 \\
\hline
\end{tabular}

CA19-9

$<35$

$>35$

Tumor location

Right-side

Left-side

Tumor size

$<50 \mathrm{~mm}$

$>50 \mathrm{~mm}$

Tumor histotype

\begin{tabular}{lllllll} 
pap/tub & $86(90.5 \%)$ & $217(96.4 \%)$ & & $76(93.8 \%)$ & $76(93.8 \%)$ & \\
\hline muc/por/sig & $9(9.5 \%)$ & $8(3.6 \%)$ & 0.052 & $5(6.2 \%)$ & $5(6.2 \%)$ & 1.000
\end{tabular}

Lymphatic

invasion

No

$38(40.0 \%)$

$131(58.2 \%)$
$65(80.2 \%) \quad 66(81.5 \%)$

$16(19.8 \%) \quad 15(18.5 \%) \quad 1.000$

$\begin{array}{llllll}52(54.7 \%) & 143(63.6 \%) & 0.168 & 48(59.3 \%) & 53(65.4 \%) & 0.517\end{array}$

\begin{tabular}{llllll}
$54(56.8 \%)$ & $171(76.0 \%)$ & $51(63.0 \%)$ & $49(60.5 \%)$ & \\
\hline $41(43.2 \%)$ & $54(24.0 \%)$ & 0.001 & $30(37.0 \%)$ & $32(39.5 \%)$ & 0.872
\end{tabular}


Yes

$57(60.0 \%)$

$94(41.8 \%)$

0.003

$45(55.6 \%)$

$49(60.5 \%)$

0.633

Venous invasion

\begin{tabular}{lllllll} 
No & $45(47.4 \%)$ & $143(63.6 \%)$ & & $41(50.6 \%)$ & $39(48.1 \%)$ & \\
\hline Yes & $50(52.6 \%)$ & $82(36.4 \%)$ & 0.009 & $40(49.4 \%)$ & $42(51.9 \%)$ & 0.875
\end{tabular}

Pathological stage

\begin{tabular}{lllllll}
\hline 0 & $9(9.5 \%)$ & $25(11.1 \%)$ & $9(11.1 \%)$ & $4(4.9 \%)$ & \\
\hline I & $16(16.8 \%)$ & $64(28.4 \%)$ & $16(19.8 \%)$ & $16(19.8 \%)$ & \\
\hline II & $32(33.7 \%)$ & $81(36.0 \%)$ & $22(27.2 \%)$ & $37(45.7 \%)$ & \\
\hline III & $38(40.0 \%)$ & $55(24.4 \%)$ & 0.026 & $34(42.0 \%)$ & $24(29.6 \%)$ & 0.060 \\
\hline $\begin{array}{l}\text { Postoperative } \\
\text { complication }\end{array}$ & & & & & & \\
\hline $\begin{array}{l}\text { Clavien-Dindo } \\
>\text { grade II }\end{array}$ & $13(13.8 \%)$ & $12(5.4 \%)$ & 0.020 & $11(13.8 \%)$ & $2(2.5 \%)$ & 0.009 \\
\end{tabular}

CRC, Colorectal cancer; CAR, C-reactive protein/albumin ratio; PSM, propensity score matching; ASAPS, American Society of Anesthesiologists physical status

CEA, carcinoembryonic antigen; CA19-9, carbohydrate antigen; pap, papillary adenocarcinoma; tub, tubular adenocarcinoma; muc, mucinous adenocarcinoma

por, poorly differentiated adenocarcinoma; sig, signet-ring cell carcinoma

Table 3 Clinicopathological parameters of CRC patients with respect to CAR at the 7th postoperative day 
Variable

\begin{tabular}{|c|c|c|c|c|c|c|}
\hline & $\begin{array}{l}\text { High CAR } \\
(>0.859)\end{array}$ & $\begin{array}{l}\text { Low CAR } \\
(<0.859)\end{array}$ & $\begin{array}{l}p- \\
\text { Value }\end{array}$ & $\begin{array}{l}\text { High CAR } \\
(>0.859)\end{array}$ & $\begin{array}{l}\text { Low CAR } \\
(<0.859)\end{array}$ & $\begin{array}{l}p- \\
\text { Value }\end{array}$ \\
\hline Sex, n & 75 & 245 & & 72 & 72 & \\
\hline Male & 52 (69.3\%) & $115(46.9 \%)$ & & 49 (68.1\%) & $54(75.0 \%)$ & \\
\hline Female & $23(30.7 \%)$ & $130(53.1 \%)$ & 0.001 & 23 (31.9\%) & $18(25.0 \%)$ & 0.460 \\
\hline Age, yr & $74.32 \pm 9.64$ & $69.94 \pm 12.02$ & 0.004 & $74.19 \pm 9.72$ & $73.44 \pm 9.17$ & 0.635 \\
\hline \multicolumn{7}{|l|}{ ASA-PS } \\
\hline 1 & $2(2.7 \%)$ & $30(12.2 \%)$ & & $2(2.8 \%)$ & $1(1.4 \%)$ & \\
\hline 2 & $41(54.7 \%)$ & 170 (69.4\%) & & 41 (56.9\%) & $42(58.3 \%)$ & \\
\hline 3 & $31(41.3 \%)$ & 45 (18.4\%) & & 29 (40.3\%) & $29(40.3 \%)$ & \\
\hline 4 & $1(1.3 \%)$ & $0(0.0 \%)$ & $<0.001$ & $0(0.0 \%)$ & $0(0.0 \%)$ & 1.000 \\
\hline \multicolumn{7}{|l|}{ CEA } \\
\hline$<5$ & $43(57.3 \%)$ & $154(62.9 \%)$ & & 41 (56.9\%) & 39 (54.2\%) & \\
\hline$>5$ & 32 (42.7\%) & 91 (37.1\%) & 0.417 & 31 (43.1\%) & 33 (45.8\%) & 0.867 \\
\hline \multicolumn{7}{|l|}{ CA19-9 } \\
\hline$<35$ & 64 (85.3\%) & 202 (82.4\%) & & $61(84.7 \%)$ & $56(77.8 \%)$ & \\
\hline$>35$ & $11(14.7 \%)$ & 43 (17.6\%) & 0.725 & $11(15.3 \%)$ & $16(22.2 \%)$ & 0.393 \\
\hline \multicolumn{7}{|l|}{ Tumor location } \\
\hline Right-side & $29(38.7 \%)$ & $96(39.2 \%)$ & & $28(38.9 \%)$ & $28(38.9 \%)$ & \\
\hline Left-side & $46(61.3 \%)$ & $149(60.8 \%)$ & 1.000 & $44(61.1 \%)$ & $44(61.1 \%)$ & 1.000 \\
\hline \multicolumn{7}{|l|}{ Tumor size } \\
\hline$<50 \mathrm{~mm}$ & 48 (64.0\%) & 177 (72.2\%) & & $46(63.9 \%)$ & $49(68.1 \%)$ & \\
\hline$>50 \mathrm{~mm}$ & 27 (36.0\%) & 68 (27.8\%) & 0.194 & $26(36.1 \%)$ & $23(31.9 \%)$ & 0.725 \\
\hline \multicolumn{7}{|l|}{ Tumor histotype } \\
\hline pap/tub & 71 (94.7\%) & 232 (94.7\%) & & 68 (94.4\%) & $69(95.8 \%)$ & \\
\hline muc/por/sig & $4(5.3 \%)$ & $13(5.3 \%)$ & 1.000 & $4(5.6 \%)$ & $3(4.2 \%)$ & 1.000 \\
\hline \multicolumn{7}{|l|}{$\begin{array}{l}\text { Lymphatic } \\
\text { invasion }\end{array}$} \\
\hline No & 35 (46.7\%) & 134 (54.7\%) & & 33 (45.8\%) & 37 (51.4\%) & \\
\hline
\end{tabular}


Yes

$40(53.3 \%)$

$111(45.3 \%)$

0.236

$39(54.2 \%)$

$35(48.6 \%)$

0.617

Venous invasion

\begin{tabular}{lllllll}
\hline No & $40(53.3 \%)$ & $148(60.4 \%)$ & & $38(52.8 \%)$ & $45(62.5 \%)$ & \\
\hline Yes & $35(46.7 \%)$ & $97(39.6 \%)$ & 0.286 & $34(47.2 \%)$ & $27(37.5 \%)$ & 0.312
\end{tabular}

Pathological stage

\begin{tabular}{|c|c|c|c|c|c|c|}
\hline 0 & $6(8.0 \%)$ & $28(11.4 \%)$ & & $6(8.3 \%)$ & $8(11.1 \%)$ & \\
\hline I & $14(18.7 \%)$ & 66 (26.9\%) & & 13 (18.1\%) & 19 (26.4\%) & \\
\hline II & $32(41.7 \%)$ & 81 (33.1\%) & & 30 (41.7\%) & 23 (31.9\%) & \\
\hline III & $23(30.7 \%)$ & $70(28.6 \%)$ & 0.297 & 23 (31.9\%) & $22(30.6 \%)$ & 0.510 \\
\hline \multicolumn{7}{|l|}{$\begin{array}{l}\text { Postoperative } \\
\text { complication }\end{array}$} \\
\hline $\begin{array}{l}\text { Clavien-Dindo } \\
>\text { grade II }\end{array}$ & $17(23.3 \%)$ & 8 (3.3\%) & $<0.001$ & $16(22.9 \%)$ & $3(4.2 \%)$ & 0.001 \\
\hline
\end{tabular}

CRC, Colorectal cancer; CAR, C-reactive protein/albumin ratio; PSM, propensity score matching; ASAPS, American Society of Anesthesiologists physical status

CEA, carcinoembryonic antigen; CA19-9, carbohydrate antigen; pap, papillary adenocarcinoma; tub, tubular adenocarcinoma; muc, mucinous adenocarcinoma

por, poorly differentiated adenocarcinoma; sig, signet-ring cell carcinoma

\section{Figures}


A

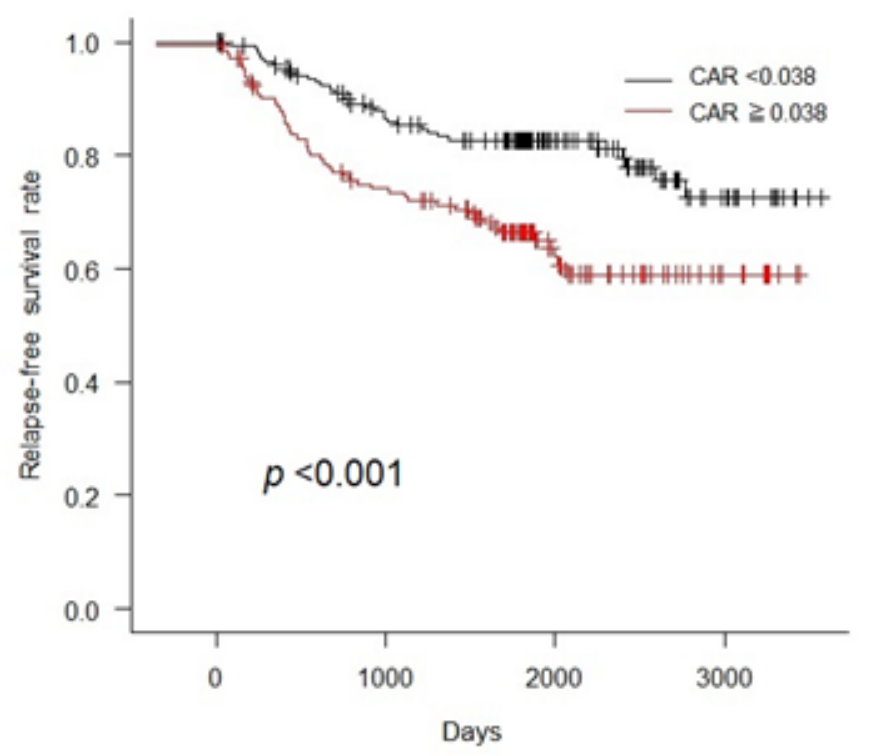

C

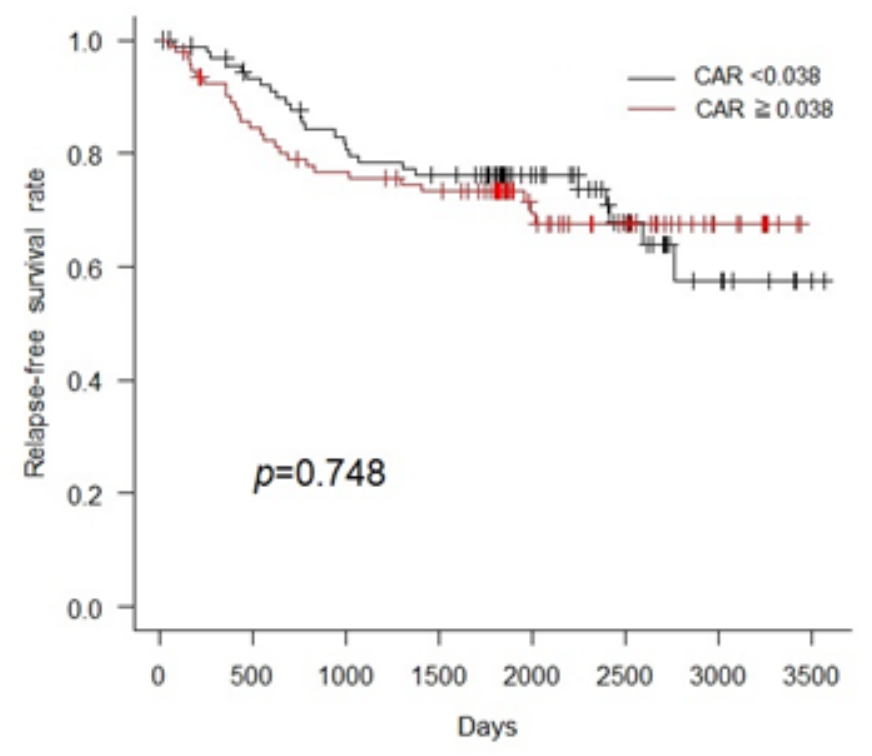

B

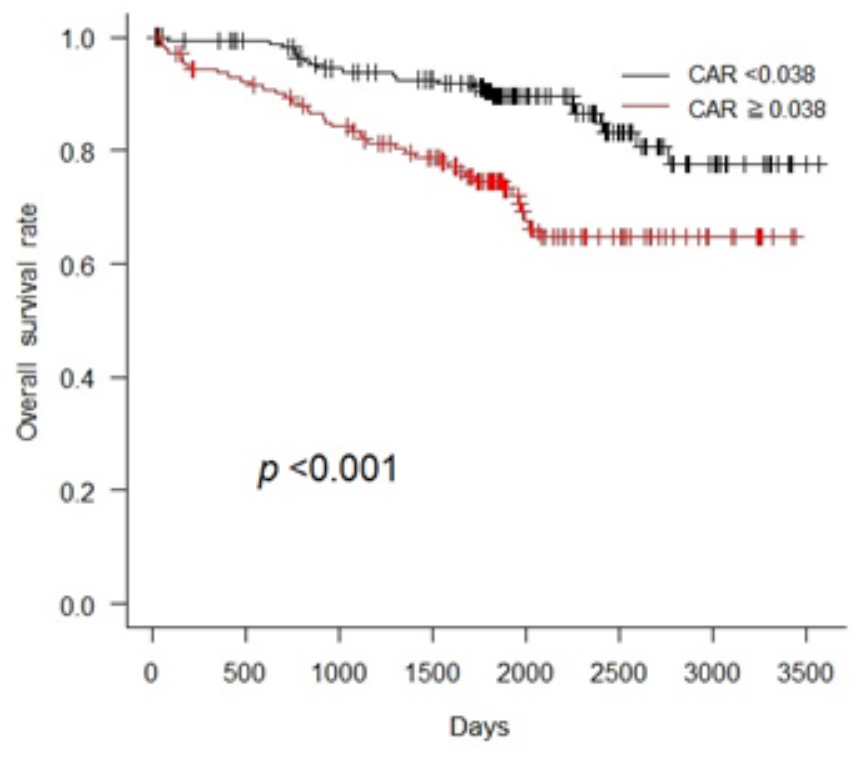

D

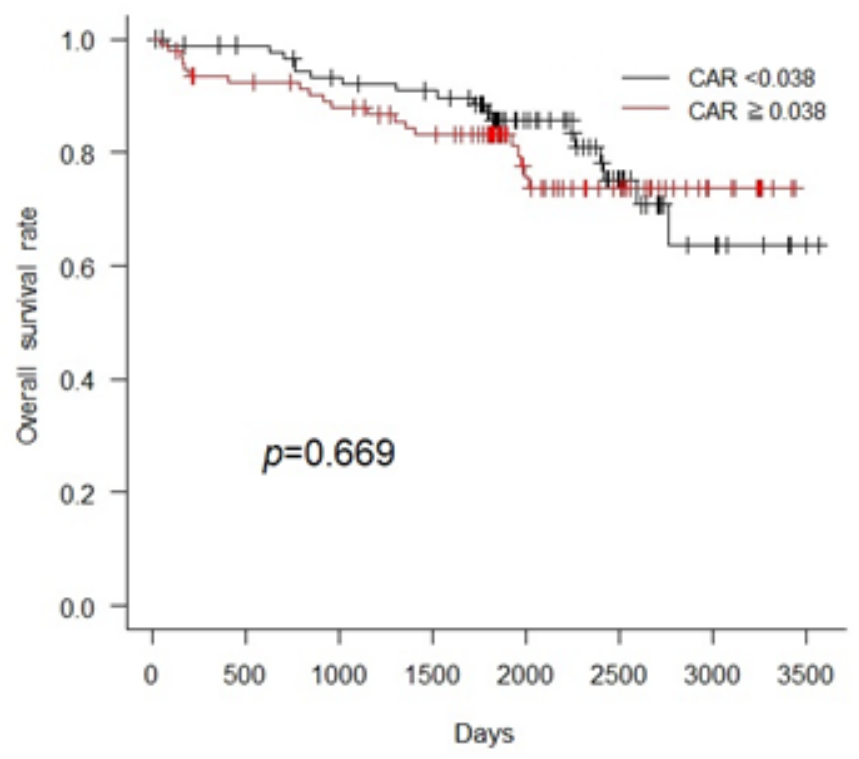

\section{Figure 1}

Analysis for preoperative CAR (A, B: RFS and OS prior to propensity score matching. C, D: RFS and OS after propensity score matching). 
A

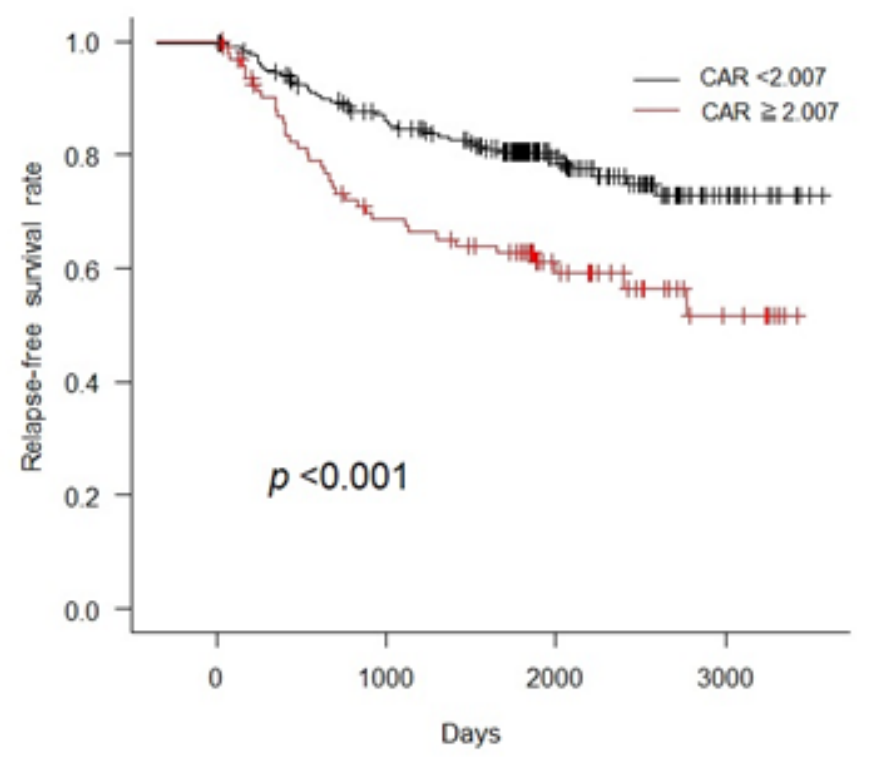

C

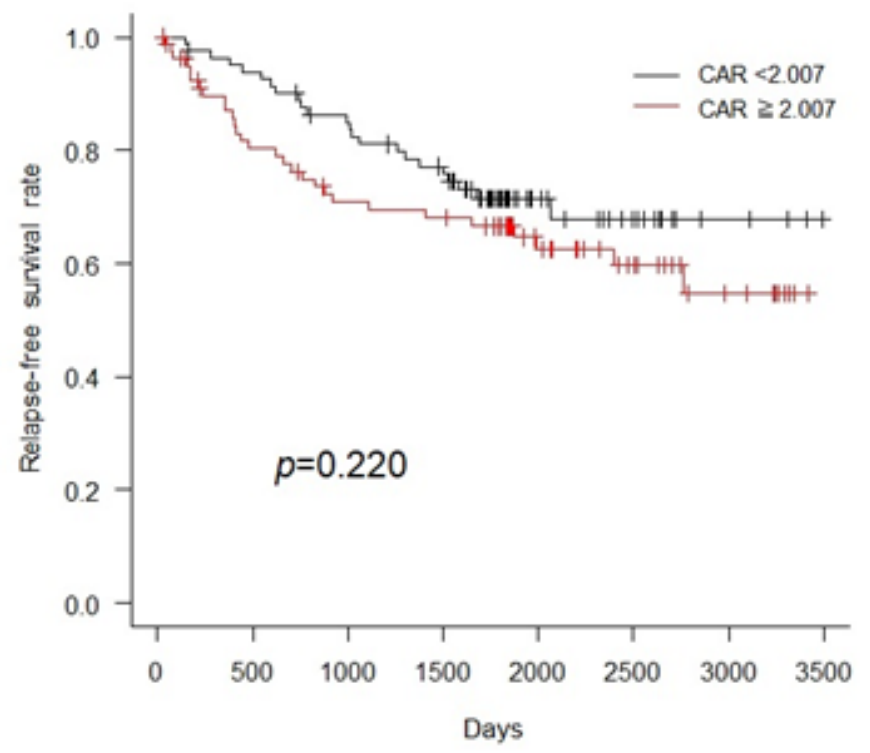

B

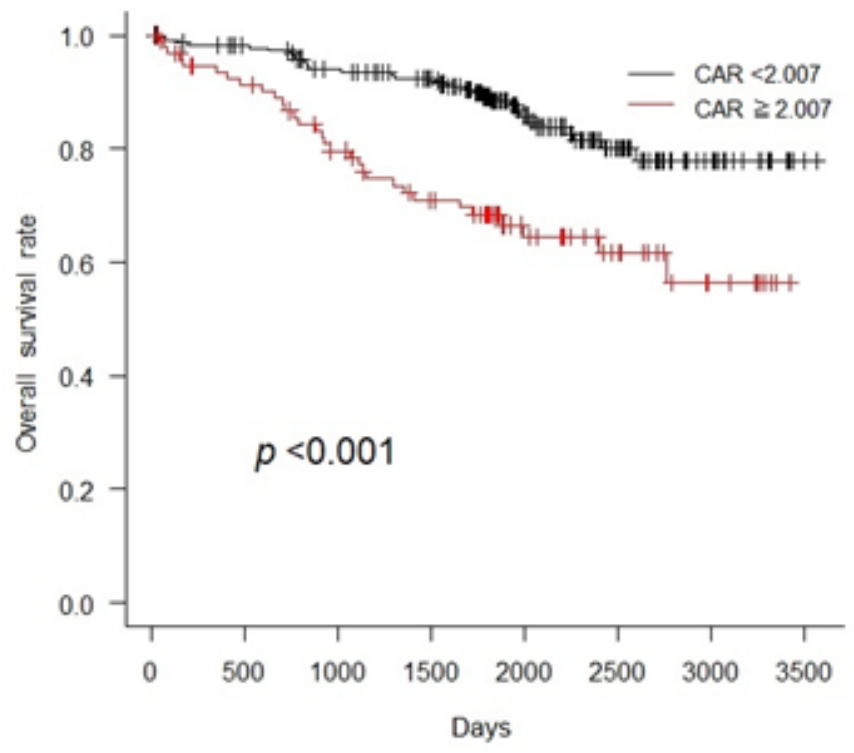

$\mathrm{D}$

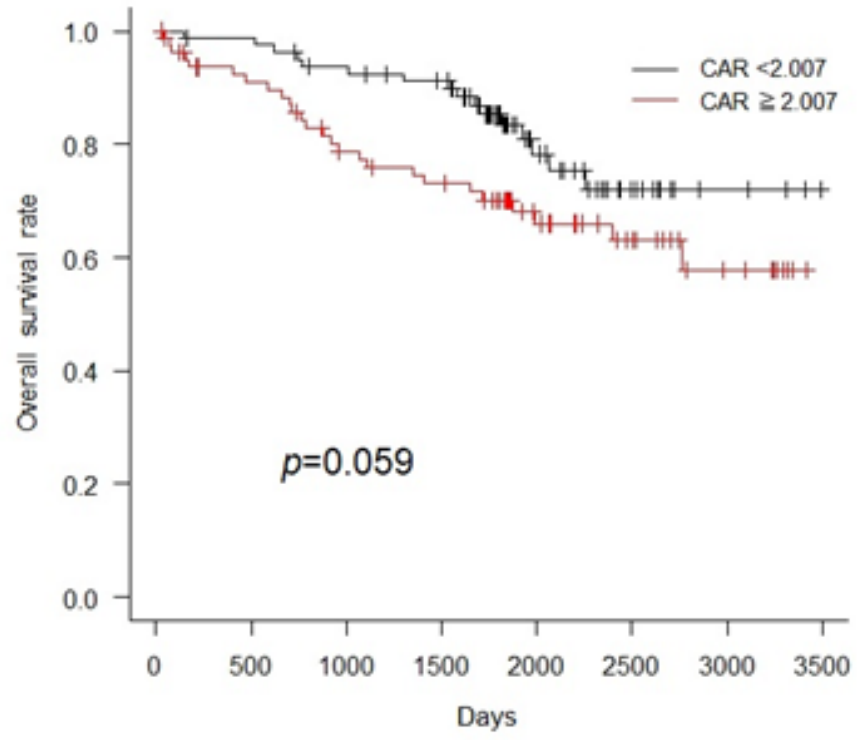

\section{Figure 2}

Analysis for CAR of POD1 (A, B: RFS and OS prior to propensity score matching. C, D: RFS and OS after propensity score matching). 
A

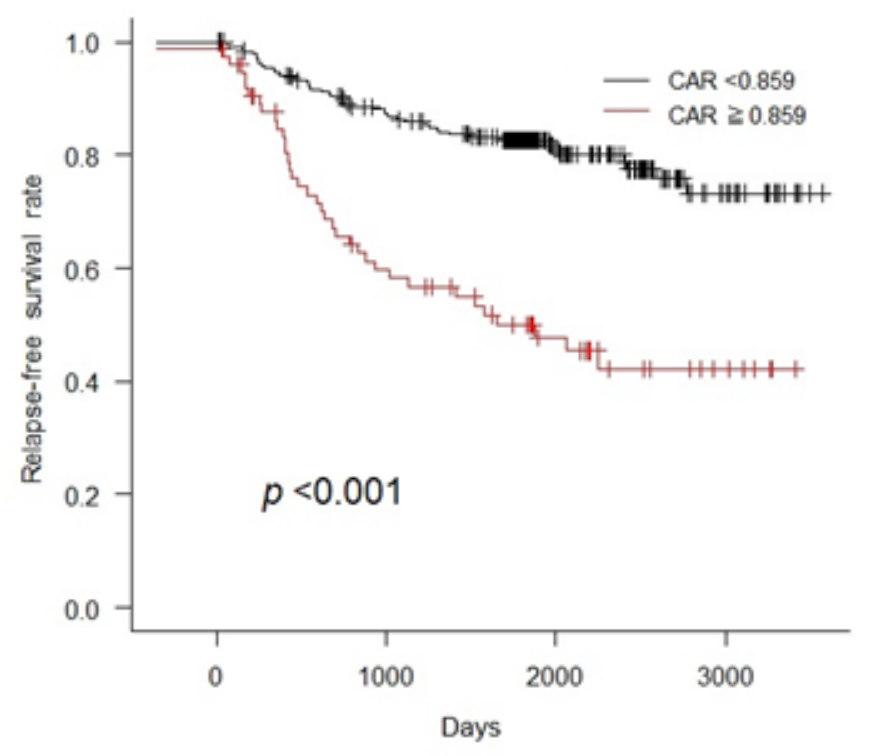

C

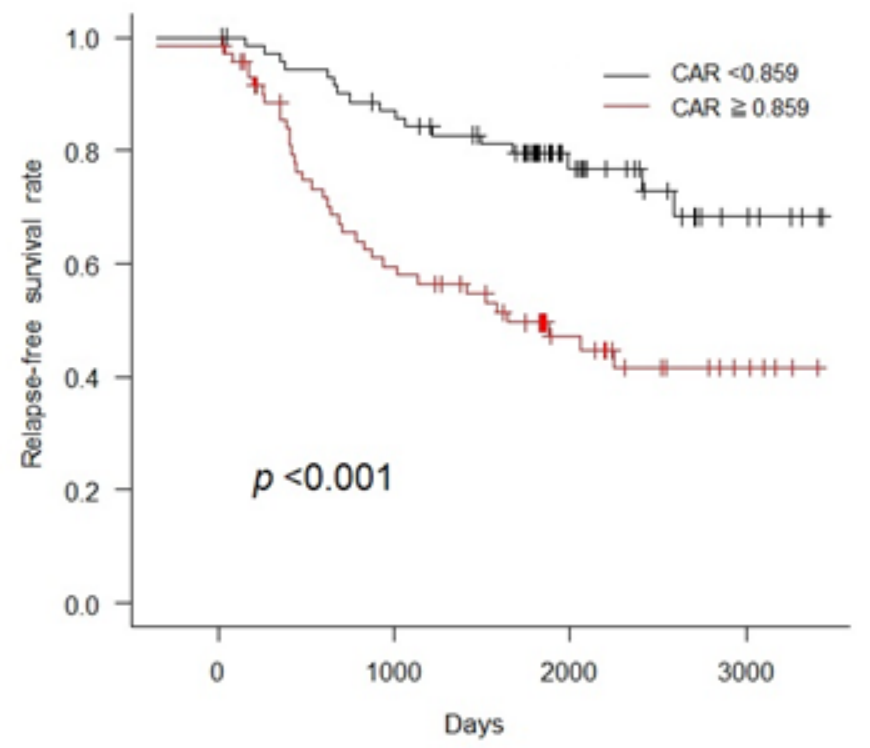

B

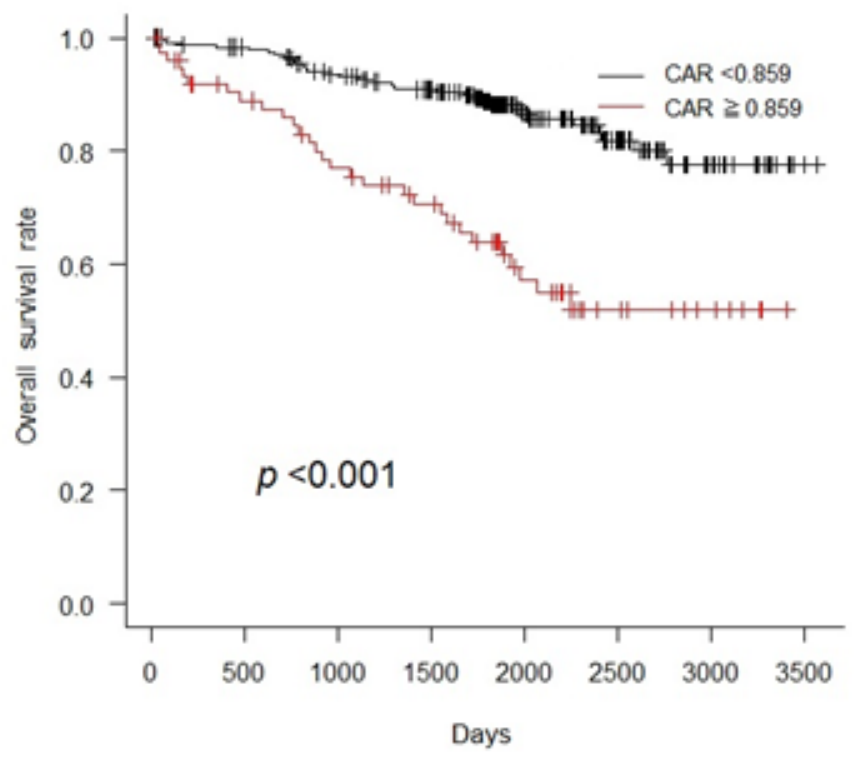

D

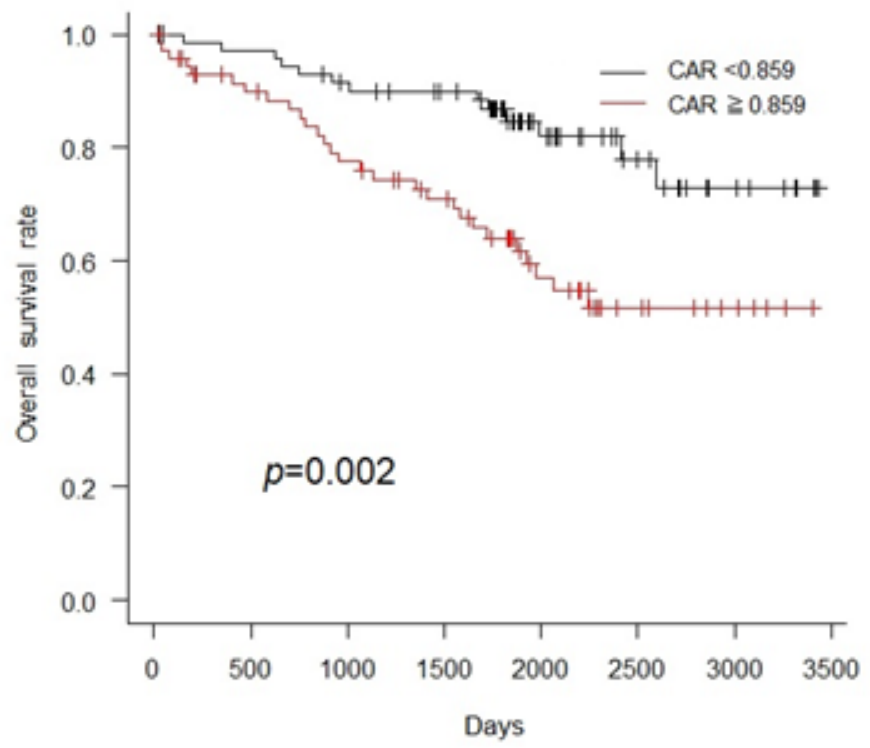

\section{Figure 3}

Analysis for CAR of POD7 (A, B: RFS and OS prior to propensity score matching. C, D: RFS and OS after propensity score matching). 
A

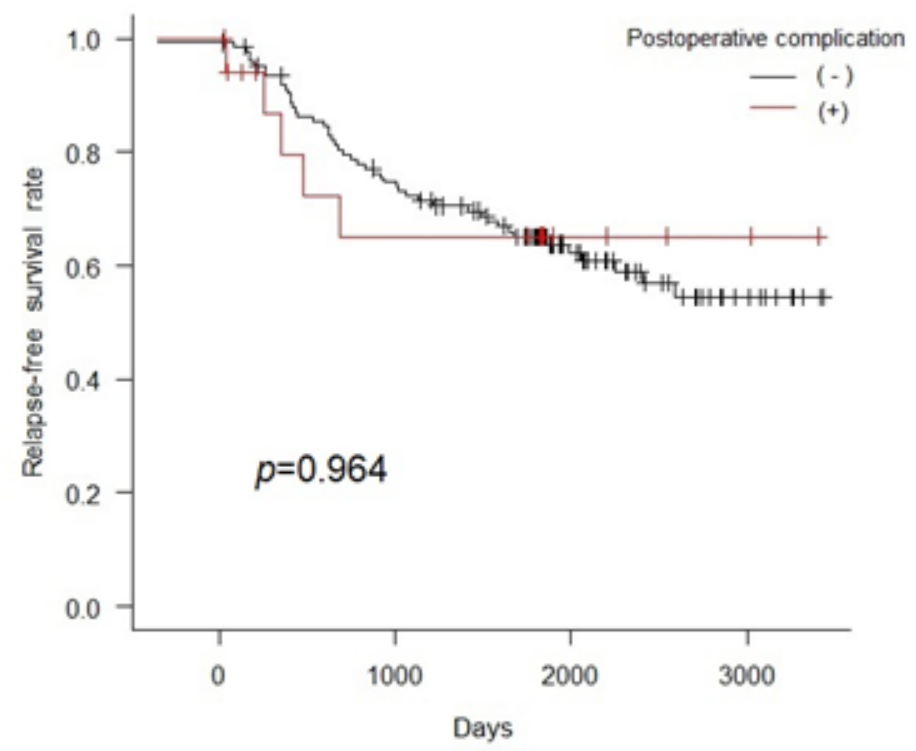

B

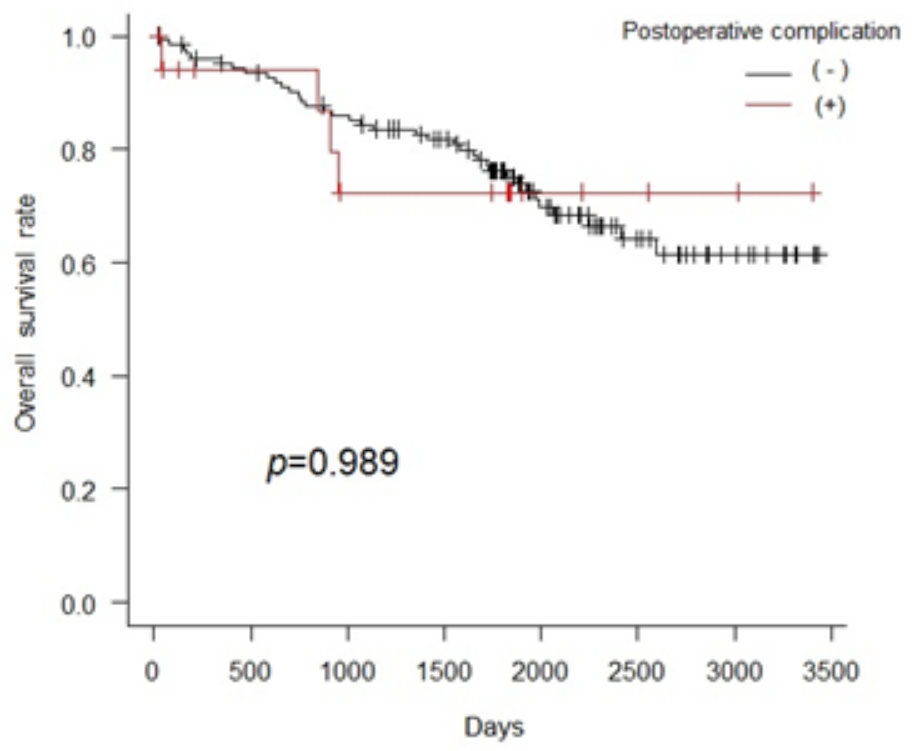

Figure 4

Comparison of RFS and OS in patients who were propensity score-matched and selected by CAR of POD7 and grouped according to the presence or absence of postoperative complication. 